\title{
Theory and Observation of Displacement Phenomena in Coadsorbed Films
}

\author{
William J. Weber and David L. Goodstein \\ Condensed Matter Physics 114-36, California Institute of Technology, Pasadena, California 91125
}

(Received 3 May 1999)

\begin{abstract}
Displacement refers to a surprising phenomenon observed in a number of adsorption systems. At low temperatures, some relatively inert gases, such as krypton $(\mathrm{Kr})$ or methane $\left(\mathrm{CH}_{4}\right)$, are found to displace molecules from preadsorbed monolayer films of a more condensable species, such as carbon tetrachloride $\left(\mathrm{CCl}_{4}\right)$, from a graphite substrate. We present a simple thermodynamic model to explain displacement and make a prediction applicable to both first-order and continuous displacement processes. We also present measurements on $\mathrm{CH}_{4} / \mathrm{CCl}_{4}$ and $\mathrm{Kr} / \mathrm{CCl}_{4}$ coadsorbed films from $77-112 \mathrm{~K}$ that verify our prediction and yield the $\mathrm{CCl}_{4}$ film spreading pressure.
\end{abstract}

PACS numbers: 82.65.Dp, 68.35.Rh, 68.45.Gd

Simultaneous adsorption of two distinct chemical species on a smooth substrate provides an arena in which questions concerning phase separation and miscibility meet quasi-two-dimensional phenomena such as first-order and critical layering transitions, commensurability, and layer melting. One class of coadsorption systems, in which two species with very different adsorption characteristics are adsorbed on graphite, displays a counterintuitive phenomenon known as displacement. $\mathrm{CH}_{4} / \mathrm{CCl}_{4}$ falls into this class. $\mathrm{CCl}_{4}$ interacts strongly enough with graphite to form thick films at room temperature [1]. Yet $\mathrm{CH}_{4}$, a relatively inert gas with a much weaker attraction to graphite, succeeds, at low temperatures, in displacing a preadsorbed monolayer film of $\mathrm{CCl}_{4}$ from graphite and adsorbing directly onto the substrate [2]. Displacement of a more condensable species by a relatively inert species is observed in a number of similar systems, such as $\mathrm{Kr} / \mathrm{C}_{6} \mathrm{H}_{12}$ [2,3], $\mathrm{Kr} / \mathrm{CCl}_{4}[4,5], \mathrm{Xe} / \mathrm{SF}_{6}$ [6], and $\mathrm{Kr} / \mathrm{SF}_{6}$ $[7,8]$. We present here a simple thermodynamic model of displacement and compare it with experimental data for the $\mathrm{Kr} / \mathrm{CCl}_{4}$ and $\mathrm{CH}_{4} / \mathrm{CCl}_{4}$ coadsorption systems.

The primary experimental evidence for displacement has come from coadsorption isotherms [2-7], which measure the film coverage of the inert gas species (species 2), as a function of its vapor pressure or chemical potential, $\delta \mu_{2}$, in the presence of the preadsorbate (species 1) film $\left(\delta \mu_{2} \equiv \mu_{2}-\mu_{2}^{0}\right.$, chemical potential measured relative to bulk saturation). Typically small preadsorbate saturated vapor pressures $\left(\approx 10^{-20}\right.$ Torr for $\mathrm{CCl}_{4}$ at $77 \mathrm{~K}$ [9]) permit accurate measurement of the species- 2 vapor pressure. Coadsorption (COAD) and single species (SS) isotherm data in Fig. 1 illustrate displacement of $\mathrm{CCl}_{4}$ from graphite by $\mathrm{CH}_{4}$ at several temperatures. Adsorption in the COAD systems is limited at low $\delta \mu_{\mathrm{CH}_{4}}$. However, at higher chemical potentials, coadsorption of $\mathrm{CH}_{4}$ proceeds much as in the SS case, with the COAD curves overlaying the SS curves very closely if the COAD coverage is multiplied by a scale factor, $F=1.48$. The interpretation here, in close analogy to the other coadsorption isotherm studies listed above, is that $\mathrm{CH}_{4}$, at high values of $\delta \mu_{\mathrm{CH}_{4}}$, is adsorbing on bare graphite as in SS adsorption, with $\mathrm{CCl}_{4}$ displaced from the substrate. Similar data for $\mathrm{Kr} / \mathrm{CCl}_{4}$ and an explanation of our experimental technique (and the scale factor, $F$ ) will be presented later in the paper.

Displacement of $\mathrm{CCl}_{4}$ by $\mathrm{CH}_{4}$ occurs in continuous fashion, without a vertical step in the COAD isotherm, at all temperatures of our study (a continuous isotherm for this system at $77 \mathrm{~K}$ was also presented in [2]). Other systems, $\mathrm{Kr} / \mathrm{CCl}_{4}$ at $79.6 \mathrm{~K}$, for example, exhibit first-order displacement [4]. Schematic isotherms in Fig. 2 illustrate first-order and continuous displacement. Evolution from first-order to continuous displacement with increasing temperature was also reported for the $\mathrm{Kr} / \mathrm{C}_{6} \mathrm{H}_{12}$, with a critical point of $80.25 \mathrm{~K}$ [2]. A model of coadsorption must be consistent with either type of displacement to describe the phenomenon successfully.

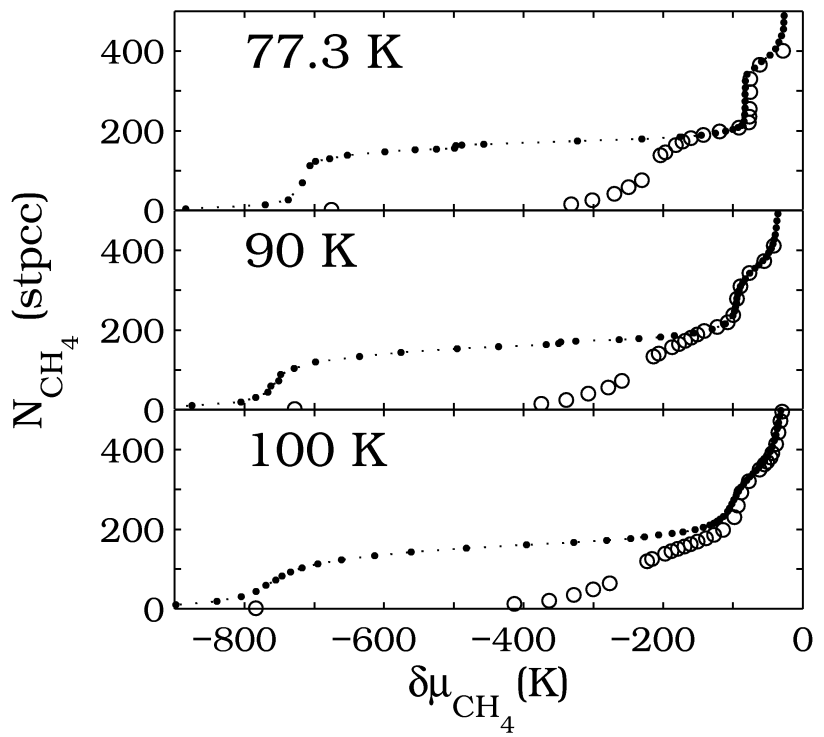

FIG. 1. $\mathrm{CH}_{4}$ isotherm data on graphite, $\mathrm{SS}(\mathbf{)})$ and COAD with $\mathrm{CCl}_{4}(\mathrm{O})$. COAD data are scaled by $F=1.48$. 1 stpcc $\equiv 2.687 \times 10^{19}$, the ideal gas number in $1 \mathrm{cc}$ at stp. Low pressure SS data at $77.3 \mathrm{~K}$ are from TD [12]. 


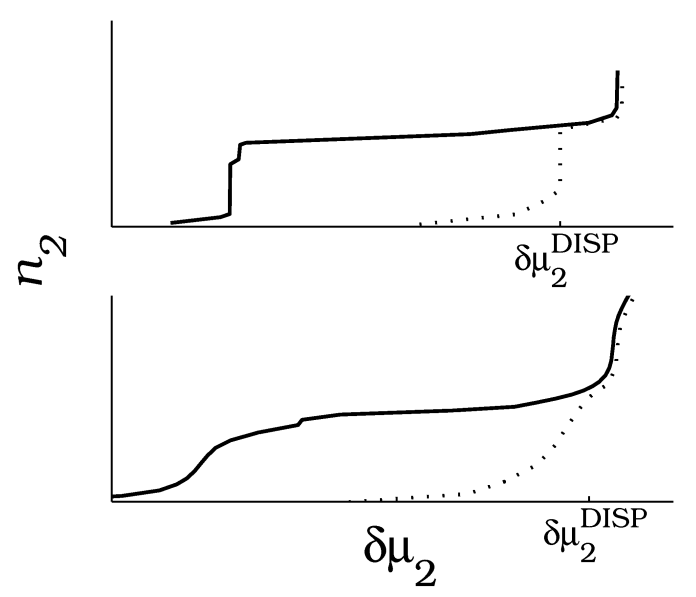

FIG. 2. Schematic isotherms illustrating first-order (top) and continuous (bottom) displacement. Species-2 film density, $n_{2}$, is plotted as a function of chemical potential, with SS adsorption and COAD curves represented by solid and dotted lines, respectively. $\delta \mu_{2}^{\text {DISP }}$ indicates the completion of displacement, accompanied by a vertical step in the case of first-order displacement.

It is the wetting behavior of the preadsorbate that makes displacement favorable and permits a simple thermodynamic picture of displacement. Previous reports have linked the preadsorbate's wetting properties to displacement $[6,10,11]$. Unlike $\mathrm{Kr}$ and $\mathrm{CH}_{4}$, which exhibit layer by layer growth into thick films on graphite [12], the preadsorbates discussed here, $\mathrm{CCl}_{4}, \mathrm{C}_{6} \mathrm{H}_{12}$, and $\mathrm{SF}_{6}$, do not fully wet graphite at the temperatures where displacement is observed $[1,3,13]$; instead, a full monolayer film coexists with its own bulk crystals. Such coexistence implies that $\mathrm{CCl}_{4}$, for example, can be moved from film to bulk phase without a change in free energy, provided it does not leave an empty space behind. In a coadsorption system, an inert gas such as $\mathrm{Kr}$ or $\mathrm{CH}_{4}$ can, at sufficiently low temperature, lower its free energy by condensing as a film on graphite. Displacement of $\mathrm{CCl}_{4}$ from the film into bulk crystals, accompanied by adsorption of the inert gas onto the bare substrate, thus lowers the total system free energy, and so, despite the stronger attraction of $\mathrm{CCl}_{4}$ to graphite, thermodynamics favors displacement. Simply put, displacement of the $\mathrm{CCl}_{4}$ film occurs not because the substrate is equally attractive to both species, but rather because the $\mathrm{CCl}_{4}$ has another equally stable phase to enter. This view of displacement is consistent with structural studies of, among others, the $\mathrm{CH}_{4} / \mathrm{CCl}_{4}$ [2] and $\mathrm{Kr} / \mathrm{CCl}_{4}$ [4] systems, which show evidence of displaced $\mathrm{CCl}_{4}$ entering bulk crystalline form.

Restricting ourselves to full monolayer films of such partially wetting preadsorbates also yields a general thermodynamic prediction for displacement. The schematic isotherms in Fig. 2 show displacement completion at $\delta \mu_{2}^{\mathrm{DISP}}$, where the curves join. For $\delta \mu_{2} \geq \delta \mu^{\mathrm{DISP}}$ in the COAD system, we assume a pure film of species 2 on graphite, with the same film density, $n_{2}\left(T, \mu_{2}\right)$, and spreading pressure, $\phi\left(T, \mu_{2}\right)$ as in the SS system,

$$
\begin{aligned}
& n_{2}^{\mathrm{COAD}}\left(T, \mu_{1}, \mu_{2}\right)=n_{2}^{\mathrm{SS}}\left(T, \mu_{2}\right), \\
& \phi^{\mathrm{COAD}}\left(T, \mu_{1}, \mu_{2}\right)=\phi^{\mathrm{SS}}\left(T, \mu_{2}\right),
\end{aligned}
$$

with temperature and the chemical potentials of both species chosen as the COAD ensemble variables. We can evaluate the spreading pressures in the SS and COAD systems, and equate them for $\delta \mu_{2}=\delta \mu_{2}^{\text {DISP }}$. For SS adsorption of 2 , the thermodynamic relation

$$
s d T-d \phi+n_{2} d \mu_{2}=0,
$$

where $s$ is the areal entropy density, can be integrated, at fixed $T$, to obtain the standard result for a single component system (as in, for instance, Ref. [14]),

$$
\phi^{\mathrm{SS}}\left(T, \delta \mu_{2}\right)=\int_{-\infty}^{\delta \mu_{2}} n_{2}^{\mathrm{SS}} d \delta \mu_{2}^{\prime} .
$$

We ignore here the spreading pressure contribution of bare graphite. For equilibrium in the COAD system,

$$
s d T-d \phi+n_{1} d \mu_{1}+n_{2} d \mu_{2}=0 .
$$

The presence of bulk crystals of species 1 simplifies evaluation of Eq. (4). Before introduction of species 2, coexistence of preadsorbate film and bulk phases dictates that $\mu_{1}$ equals the bulk saturated value, $\mu_{1}^{0}(T)$, and that the film has a "saturated" spreading pressure, $\phi_{1}^{0}(T)$. As species 2 is added at constant $T$, assuming the presence of pure bulk species 1 fixes $\mu_{1}=\mu_{1}^{0}$, allowing calculation of $\phi^{\mathrm{COAD}}$ by simple integration over $\mu_{2}$,

$\phi^{\mathrm{COAD}}\left(T, \delta \mu_{2}\right)=\phi_{1}^{0}(T)+\int_{-\infty}^{\delta \mu_{2}} n_{2}^{\mathrm{COAD}} d \delta \mu_{2}^{\prime}$.

For $\delta \mu_{2}=\delta \mu_{2}^{\text {DISP }}$, displacement is complete, and species 2 is adsorbed on bare graphite. Equations (3) and (5) thus refer to the same state of matter, with equal spreading pressures. This equality can be written

$$
\int_{-\infty}^{\delta \mu_{2}^{\text {DISP }}}\left(n_{2}^{\mathrm{SS}}-n_{2}^{\mathrm{COAD}}\right) d \delta \mu_{2}^{\prime}=\phi_{1}^{0}(T) .
$$

The quantity on the left side of Eq. (6) is simply the area between COAD and SS isotherm curves, as sketched in Fig. 2. $\phi_{1}^{0}(T)\left(=\phi_{\mathrm{CCl}_{4}}^{0}\right.$ in our experiment $)$ is a property of the preadsorbate, independent of the inert gas species, and so the measured quantity on the left side should be equal for two COAD systems using the same preadsorbate, such as $\mathrm{CH}_{4} / \mathrm{CCl}_{4}$ and $\mathrm{Kr} / \mathrm{CCl}_{4}$. The small vapor pressure of $\mathrm{CCl}_{4}$ prevents direct measurement of $\phi_{\mathrm{CCl}_{4}}^{0}(T)$. However, isotherm data for the two coadsorption systems can test this model and measure $\phi_{\mathrm{CCl}_{4}}^{0}(T)$.

Equation (6) applies to both continuous and first-order displacement. In a first-order displacement transition, two distinct phases coexist with the same spreading pressure, at $\delta \mu_{2}^{\text {DISP }}(T)$. One phase is the preadsorbate film, perhaps with some quantity of species 2 mixed in or absorbed on top, and the other consists of self-condensed 
islands of the inert gas species on bare graphite, growing at $\delta \mu_{2}=\delta \mu_{2}^{\text {DISP }}$ as species 2 is added. For the special case where $n_{2}^{\mathrm{COAD}}=0$ for $\delta \mu_{2}<\delta \mu_{2}^{\mathrm{DISP}}$, Eq. (6) is equivalent to the result of Menaucourt and Bockel, who considered first-order coexistence between pure films of each species, with a partially wetting preadsorbate [6]. However, Eq. (6) is considerably more general, also applicable when species 2 is partially or totally soluble in the preadsorbate film or adsorbs on top of it. In a fully continuous displacement, the coadsorbed film is a single mixed phase, in which the species- 2 concentration grows from zero at low $\delta \mu_{2}$ to $100 \%$ at $\delta \mu_{2}^{\mathrm{DISP}}(T)$. Thermodynamics alone does not reveal the structure of the coadsorbed film prior to displacement or predict which type of displacement should occur. However, Eq. (6) expresses the general condition of equilibrium thermodynamics, applicable to any system where displacement of a saturated preadsorbate film ends with a pure film of the second species. Our measurements of $\mathrm{CH}_{4} / \mathrm{CCl}_{4}$ and $\mathrm{Kr} / \mathrm{CCl}_{4}$ coadsorption test this thermodynamic description of displacement.

Coadsorption experiments were carried out in a graphite calorimetry cell, which was used simultaneously for a film heat capacity study [15]. The cell consists of $100 \mathrm{cc}$ of exfoliated graphite foam [16] with a $\sqrt{3} \times \sqrt{3}$ monolayer coverage, $N_{\sqrt{3}}$, of 165 stpcc. After transfer into our gas handling system, the preadsorbate, $\mathrm{CCl}_{4}$ [17], was purified of volatile contaminants by successive pumpouts at $77 \mathrm{~K}$ [4]. To form a uniform and complete $\mathrm{CCl}_{4}$ monolayer film, the cell was intentionally overfilled (430 stpcc of $\mathrm{CCl}_{4}$, roughly 5 times the monolayer quantity) at room temperature and then slowly cooled to $70 \mathrm{~K}$. $\mathrm{CH}_{4} / \mathrm{CCl}_{4}$ and $\mathrm{Kr} / \mathrm{CCl}_{4}$ coadsorbed films were grown by adding small doses of the inert gas species, typically less than $10 \%$ of a monolayer. For each cell filling, a dynamic measurement of film vapor pressure was made while scanning over a range of temperatures, allowing a volumetric calculation of film coverage, $N_{2}^{\mathrm{COAD}}\left(T, \delta \mu_{2}\right)$. Coadsorption isotherms were then reconstructed using the complete data set of all cell fillings. Single species adsorption isotherms of $\mathrm{Kr}$ and $\mathrm{CH}_{4}$ were measured at various fixed temperatures using a standard volumetric technique, or taken from published data measured using the same cryostat $[18,19]$. At lower temperatures, where film condensation pressures fall below the accurate range of our gauge, SS isotherm data from Thomy and Duval (TD), as read from published graphs [12], are used. All data were corrected for thermal transpiration [20], and a correction for the finite film-gas equilibration time was performed on the dynamic data. While equilibration was slowed on crossing $\delta \mu_{2}^{\mathrm{DISP}}(T)$, the coverage was observed to change reversibly; adsorption as the system was cooled across the line of displacement completion was followed by the same amount of desorption upon warming. A COAD isotherm for $\mathrm{CH}_{4} / \mathrm{CCl}_{4}$ was measured at a fixed temperature of $77 \mathrm{~K}$ and confirmed the spreading pressure obtained by our dynamic technique.
Details of our apparatus are provided in Ref. [18], and a complete explanation of our experiment and analysis will be given in a subsequent publication [15].

Figures 1 and 3 display coadsorbed film isotherm data for $\mathrm{CH}_{4} / \mathrm{CCl}_{4}$ and $\mathrm{Kr} / \mathrm{CCl}_{4}$, plotted with the corresponding single species data. The inert gas species coverage for each COAD system, $N_{2}^{\mathrm{COAD}}$, has been multiplied by a scale factor, $F$, chosen to allow overlap with the SS coverages at $\delta \mu_{2}$, where displacement is complete. The value of $F$ for the data presented here, measured with a single filling of $\mathrm{CCl}_{4}$, was found to be $1.48 \pm 0.02$ for the $\mathrm{CH}_{4} / \mathrm{CCl}_{4}$ and $1.60 \pm 0.02$ for $\mathrm{Kr} / \mathrm{CCl}_{4}$. Our need for a scale factor, $F>1$, to overlap the COAD and SS data reflects the reduction in the total coverage of species 2 in the COAD system (relative to the SS case) after displacement, as observed in every coadsorption isotherm study referenced above. While displaced $\mathrm{CCl}_{4}$ is shown to make bulk crystals, it has been suggested that these crystals are relatively flat and thus take up a sizable portion of the graphite surface [2]. This appears consistent with the low $\mathrm{CCl}_{4}$ vapor pressure, which may prevent true equilibration in the sizes and positions of crystals on experimental time scales. $F^{-1}$ represents the fraction of the surface remaining for adsorption by species 2 after displacement. Disequilibrium may also explain the variation we find in $F$, not only between the two coadsorption systems, but also for the same system grown twice on the same filling of $\mathrm{CCl}_{4}$. However, for a data set from a single coadsorbed film grown in small doses, a single value of $F$ produces good overlap with the single species data across our temperature range, as seen in Figs. 1 and 3. Also, the spreading pressures and chemical potentials at which displacement is completed are reproducible within

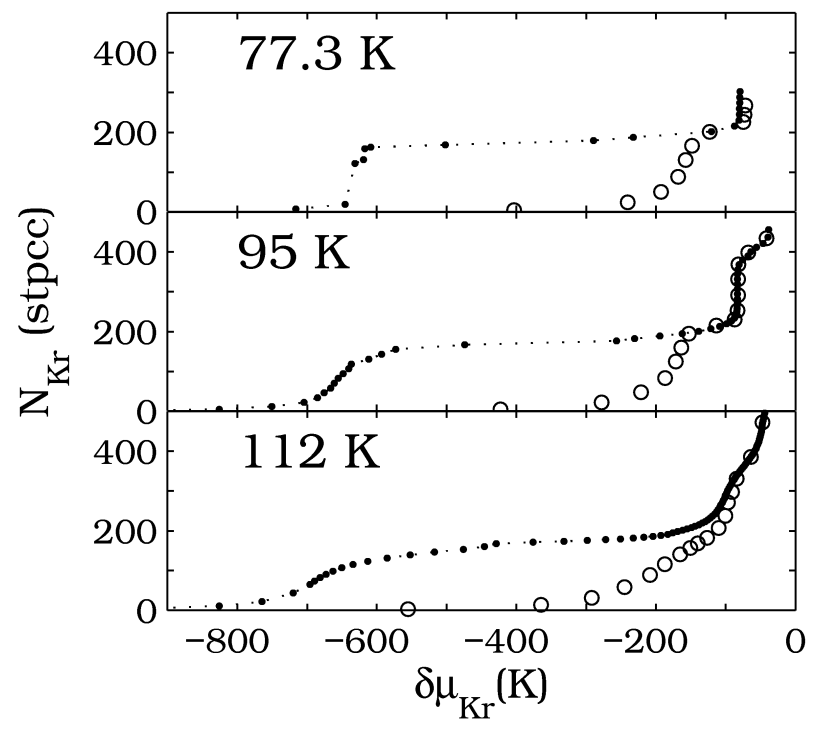

FIG. 3. Kr isotherm data on graphite, SS (-) and COAD with $\mathrm{CCl}_{4}(\mathrm{O})$. COAD data are scaled by $F=1.60$. Low pressure SS data at $77.3 \mathrm{~K}$ are from TD [12]. 


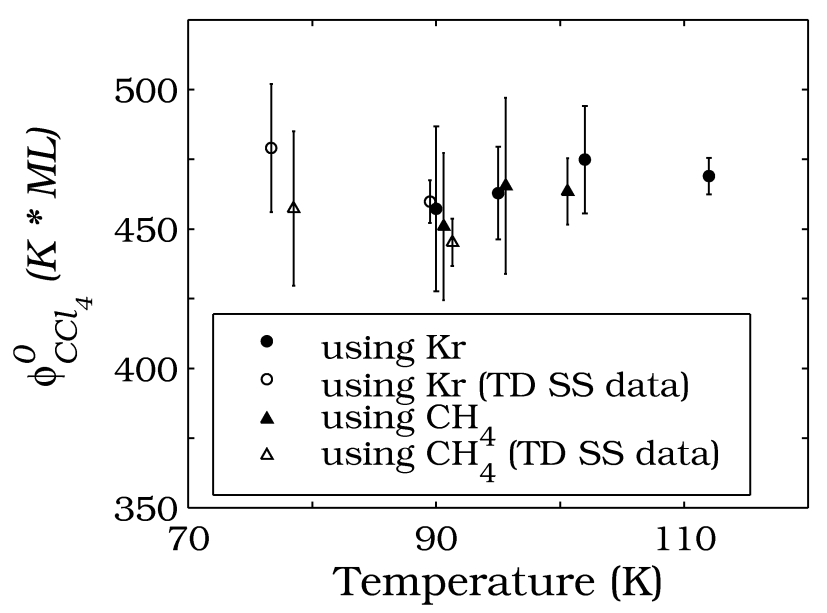

FIG. 4. $\phi_{\mathrm{CCl}_{4}}^{0}$, calculated from $\mathrm{Kr} / \mathrm{CCl}_{4}$ and $\mathrm{CH}_{4} / \mathrm{CCl}_{4}$ data. "ML" refers to the $\sqrt{3} \times \sqrt{3}$ monolayer density. Low pressure single species data from [12] have been used to calculate $\phi$ for the "TD SS" data points at 77.3 and $90.1 \mathrm{~K}$. Data points taken at the same temperature have been slightly separated in order to show the individual points and error bars.

experimental uncertainties for data sets measured with different $\mathrm{CCl}_{4}$ fillings and different values of $F$.

The data in Figs. 1 and 3 can be integrated, as in Eq. (6), to give the $\mathrm{CCl}_{4}$ spreading pressure and to compare directly the two coadsorption systems. Film densities, in units of $\sqrt{3} \times \sqrt{3}$ monolayers, ML, are simply $n_{2}^{\mathrm{SS}}=N_{2}^{\mathrm{SS}} / N_{\sqrt{3}}$ and $n_{2}^{\mathrm{COAD}}=\left(F * N_{2}^{\mathrm{COAD}}\right) / N_{\sqrt{3}}$. We perform the integral on the left side of Eq. (6) numerically, by evaluating the area of the polygon formed by the SS and COAD isotherm data points. Results of this calculation, for $\phi_{\mathrm{CCl}_{4}}^{0}$ using both $\mathrm{Kr}$ and $\mathrm{CH}_{4}$, are displayed in Fig. 4. The dominant source of error is uncertainty in the chemical potential measurements at low adsorption pressures. The agreement between the $\mathrm{CH}_{4} / \mathrm{CCl}_{4}$ and $\mathrm{Kr} / \mathrm{CCl}_{4}$ results confirms our thermodynamic model; the measured value of $\phi_{\mathrm{CCl}_{4}}^{0}(T)$ is the same whether $\mathrm{Kr}$ or $\mathrm{CH}_{4}$ serves as the displacing inert gas species.

We conclude with a few comments on the data presented here. The estimate of $\phi_{\mathrm{CCl}_{4}}^{0}$ from displacement data is independent of the inert gas species used, as expected, but also exhibits no temperature dependence (see Fig. 4). A truly temperature independent $\phi_{\mathrm{CCl}_{4}}^{0}$ would imply that the phase transition from monolayer film to bulk solid for $\mathrm{CCl}_{4}$ has no latent heat. Assuming temperature independence, the data are consistent with

$$
\phi_{\mathrm{CCl}_{4}}^{0}=465 \pm 15(\mathrm{KML}) .
$$

The $\mathrm{CCl}_{4}$ spreading pressure measurement is remarkably insensitive to the wide variety in the coadsorption isotherms used to obtain it, including a nearly vertical dis- placement step for $\mathrm{Kr}$ at $77 \mathrm{~K}$ and clearly continuous displacement for both species at higher temperatures, where displacement is not complete until well into second layer film growth (see Figs. 1 and 3). The steep but finite slope in the COAD isotherm for $\mathrm{Kr}$ at $77 \mathrm{~K}$ may be consistent with earlier studies finding first-order displacement [4] if bulk $\mathrm{CCl}_{4}$ is in microcrystals or capillary condensate, as observed previously for inert gas adsorption in our exfoliated graphite sample [21]. Continuous displacement isotherms were seen before for $\mathrm{CH}_{4} / \mathrm{CCl}_{4}$, although neutron diffraction studies actually revealed separate $\mathrm{CCl}_{4}$ and $\mathrm{CH}_{4}$ solid film domains [2]. While these scattering results conflict with the picture of a continuously mixed film, our data also show continuous displacement, for both species over a wide range of temperatures, and are consistent with the thermodynamic requirements expressed in this paper. The model presented and verified here provides a thermodynamic basis for further investigation into the possible phases, pure and mixed, formed in such coadsorption systems. Our calorimetry study, to be published, will probe the complete coadsorption phase diagram for $\mathrm{CH}_{4} / \mathrm{CCl}_{4}$ and $\mathrm{Kr} / \mathrm{CCl}_{4}$.

[1] P. W. Stephens and M.F. Huth, Phys. Rev. B 32, 1661 (1985).

[2] N. Dupont-Pavlovsky et al., Surf. Sci. 317, 388 (1994).

[3] A. Razafitianamaharavo, N. Dupont-Pavlovsky, and A. Thomy, J. Phys. (Paris) 51, 91 (1990).

[4] M. Abdelmoula et al., Surf. Sci. 272, 167 (1992).

[5] H. Asada, S. Doi, and H. Kawano, Surf. Sci. Lett. 273, L403 (1992).

[6] J. Menaucourt and C. Bockel, J. Phys. (Paris) 51, 1987 (1990).

[7] M. Bouchdoug, J. Menaucourt, and A. Thomy, J. Phys. (Paris) 47, 1797 (1986).

[8] M. A. Castro and R. K. Thomas, Surf. Sci. 399, 212 (1998).

[9] M. Abdelmoula et al., Surf. Sci. 274, 129 (1992).

[10] J. Menaucourt, J. Phys. I (France) 3, 1201 (1993).

[11] B. Mutaftschiev, Phys. Rev. B 40, 779 (1989).

[12] A. Thomy and X. Duval, J. Chim. Phys. 67, 1101 (1970).

[13] C. Marti et al., J. Phys. (Paris) 47, 1517 (1986).

[14] W. A. Steele, The Interaction of Gases with Solid Surfaces (Pergamon Press, Oxford, 1974).

[15] W. J. Weber and D. L. Goodstein (to be published).

[16] Courtesy of Union Carbide.

[17] $\mathrm{CCl}_{4}$ obtained from Aldrich-Sigma at $99.9+\%$ purity.

[18] M. J. Lysek et al., Phys. Rev. B 47, 7389 (1992); M. J. Lysek et al., Rev. Sci. Instrum. 63, 5750 (1992).

[19] P. K. Day, Ph.D. thesis, California Institute of Technology, 1993.

[20] T. Takaishi and Y. Sensui, Trans. Faraday Soc. 59, 2503 (1963).

[21] M. Lysek et al., Langmuir 9, 1040 (1993). 